\title{
X-ray emission from a metal depleted accretion shock onto the classical T Tauri star TW Hya
}

\author{
B. Stelzer ${ }^{1,2}$ and J. H. M. M. Schmitt ${ }^{3}$ \\ 1 INAF - Osservatorio Astronomico di Palermo, Piazza del Parlamento 1, 90134 Palermo, Italy \\ 2 Max-Planck-Institut für extraterrestrische Physik, Postfach 1312, 85741 Garching, Germany \\ ${ }^{3}$ Hamburger Sternwarte, Gojenbergsweg 112, 21029 Hamburg, Germany
}

Received 12 February 2003 / Accepted 2 February 2004

\begin{abstract}
We present the X-ray spectrum of TW Hya observed at high and intermediate spectral resolution with the Reflection Grating Spectrometer (RGS) and the European Photon Imaging Camera (EPIC) onboard the XMM-Newton satellite. TW Hya is the first classical T Tauri star for which simultaneous X-ray data with both high spectral resolution and high sensitivity were obtained, thus allowing to probe the X-ray emission properties of stars in the early pre-main sequence phase. Despite TW Hya's high X-ray luminosity in excess of $10^{30} \mathrm{erg} / \mathrm{s}$ its X-ray spectrum is dominated by emission lines from rather cool plasma $(T \approx 3 \mathrm{MK})$, and only little emission measure is present at high temperatures $(T \approx 10 \mathrm{MK})$. We determine photon fluxes for the emission lines in the high resolution spectrum, confirming the earlier result from Chandra that the predominant emission is from neon and oxygen, with comparatively weak iron lines. Further, the line ratios of He-like triplets of nitrogen, oxygen and neon require densities of $n_{\mathrm{e}} \sim 10^{13} \mathrm{~cm}^{-3}$, about two orders of magnitude higher than for any other star observed so far at high spectral resolution. Finally, we find that nearly all metals are underabundant with respect to solar abundances, while the abundances of nitrogen and neon are enhanced. The high plasma density, the (comparatively) low temperature, and peculiar chemical abundances in the X-ray emitting region on TW Hya are untypical for stellar coronae. An alternative X-ray production mechanism is therefore called for and a natural explanation is an accretion column depleted of grain forming elements. The metal depletion could be either due to the original molecular cloud that formed TW Hya or due to a settling of dust in the circumstellar disk of TW Hya.
\end{abstract}

Key words. X-rays: stars - stars: individual: TW Hya - stars: pre-main sequence - stars: coronae - stars: activity - accretion

\section{Introduction}

T Tauri stars (TTS) are late-type pre-main sequence (PMS) stars, usually found in or near star formation regions. The two subclasses of TTS, termed "classical" and "weak-line", are distinguished by the strength of their $\mathrm{H}_{\alpha}$ emission. This empirical distinction most likely also reflects different evolutionary states: Most of the "classical" TTS (cTTS), i.e., those with strong $\mathrm{H}_{\alpha}$ emission, show signatures of circumstellar matter (e.g. IR and UV excesses) accreting onto the central star, while in contrast, "weak-line" TTS (wTTS) show no signs for the presence of disks which presumably have been lost. A detailed description of early stellar evolution and the observable stellar properties related with the different phases can be found in Feigelson \& Montmerle (1999).

X-ray emission from TTS was first detected with the Einstein satellite (e.g. Feigelson \& DeCampli 1981; Walter \& Kuhi 1981; Montmerle et al. 1983). It was soon recognized that $\mathrm{X}$-ray observations provide an efficient means of discovering

Send offprint requests to: $\mathrm{B}$. Stelzer,

e-mail: stelzer@astropa.unipa.it
wTTS which can easily escape other identification methods because of their lack of conspicuous features in the optical or near-infrared. Extensive X-ray studies of a variety of star forming regions were undertaken with ROSAT. In particular the spatial completeness of the ROSAT All-Sky Survey has resulted in a large number of newly identified PMS stars (e.g. Neuhäuser et al. 1995; Alcalá et al. 1995; Wichmann et al. 1996).

The origin of the X-ray emission from wTTS is commonly interpreted in terms of scaled-up solar-type magnetic activity, but the situation is less clear for their accreting counterparts, the cTTS. While nothing should prevent the formation of magnetic fields and ensuing coronae in cTTS, X-rays could alternatively also be produced above the hot spot where a magnetically funnelled accretion flow impacts on the surface of the star (Lamzin 1999). A distinction between a corona or an accretion funnel as the site of X-ray emission in cTTS requires a precise assessment of the physical conditions in the X-ray emitting region. With the lower resolution X-ray data available from Einstein- and ROSAT-observations of both cTTS and wTTS it was impossible to adequately characterize the emission site, although some differences between the two types of TTS were 
indicated by statistical comparisons of their emission level and variability (see e.g. Neuhäuser et al. 1995; Stelzer et al. 2000; Stelzer \& Neuhäuser 2001a).

With the advent of high-resolution X-ray spectroscopy with the grating spectrometers onboard the XMM-Newton and Chandra observatories this situation has fundamentally changed. The reflection gratings onboard XMM-Newton and the transmission gratings onboard Chandra have spectral resolutions sufficiently large to resolve individual emission lines in the soft X-ray range. These emission lines and in particular the analysis of their relative strengths provide unprecedented tools for the plasma diagnostics of the outer envelopes of stars and allow an assessment of the temperature structure, density, and chemical abundances in the X-ray emitting region.

The high signal-to-noise ratio (SNR) required for a meaningful analysis of high-resolution X-ray spectra clearly favors the study of the nearest star forming regions. The nearby TTS TW Hya (located at a distance of only $\sim 57 \mathrm{pc}$; Wichmann et al. 1998) was first thought to be an isolated cTTS (Rucinski \& Krautter 1983), but later five more stars were found in the same region and shown to be members of a physical cluster of young stars dominated by TW Hya. The whole group is now known as the TW Hydra association (TWA; Kastner et al. 1997), which is currently known to host about 20 young stars (youth being probed by Li absorption), most of which - in contrast to TW Hya itself - are wTTS.

The first - and so far only - example of a highresolution X-ray spectrum of an accreting PMS star was a Chandra/HETGS spectrum of TW Hya presented by Kastner et al. (2002). Interestingly, flux ratios of the OVII triplett lines indicated densities much higher than found in any stellar corona before, and consequently Kastner et al. (2002) called into question the solar-stellar analogy for TW Hya and cTTS in general. About a year after the Chandra observation TW Hya was observed by XMM-Newton, resulting in a higher SNR grating spectrum with the XMM-Newton Reflection Grating Spectrometer (RGS) as well as a simultaneously measured medium-resolution CCD spectrum with high sensitivity over a broad energy range $(E=0.3-10 \mathrm{keV})$ from the European Photon Imaging Camera (EPIC). The purpose of this paper is to present the results of this $30 \mathrm{ks} X M M-N e w t o n$ observation and properly discuss their implications in the context of X-ray emission from TTS.

\section{Observations}

TW Hya was observed with XMM-Newton on July 9, 2001 for a duration of $30 \mathrm{ks}$ (Obs-ID 0112880201) with the RGS as prime instrument. The observations were performed in fullframe mode employing the medium filter for both the MOS and pn cameras of EPIC. Because of the optical brightness of TW Hya ( $V=11 \mathrm{mag}$ ) the Optical Monitor (OM) was in blocked position so that no concurrent optical data is available. The observing log for all X-ray instruments onboard $X M M-N e w t o n$ is given in Table 1 .

TW Hya is by far the brightest X-ray source and the only known member of the TW Hya association in this EPIC field. By inspecting the time-resolved background emission we
Table 1. Observing log for the XMM-Newton observation of TW Hya on July 9, 2001. "Start" and "Stop" refer to start and end of exposures.

\begin{tabular}{lccccc}
\hline \hline \multirow{2}{*}{ Instr. } & \multicolumn{2}{c}{ UT [hh:mm:ss] } & \multicolumn{2}{c}{ JD - 2452099 } & Expo \\
& Start & Stop & Start & Stop & {$[\mathrm{ks}]$} \\
\hline pn & $06: 35: 38$ & $13: 57: 31$ & 0.774306 & 1.081250 & 26.52 \\
MOS & $05: 58: 02$ & $14: 01: 48$ & 0.748611 & 1.084028 & 28.98 \\
RGS & $05: 51: 39$ & $14: 05: 15$ & 0.743750 & 1.086806 & 29.64 \\
\hline
\end{tabular}

verified that the background was low and showed only little time variability without any significant solar-activity related contamination, so that the full observing time provided data useful for analysis. The pn data shows slight evidence for pileup. Therefore, we base our analysis on the MOS detectors, which furthermore provide a better energy resolution.

We constructed an X-ray light curve of TW Hya by binning all photons from a circular area around the target position. We used an extraction radius of $40^{\prime \prime}$ including about $90 \%$ of the source photons. For the background subtraction we used a source-free circle of the same area located near TW Hya. Background subtracted MOS lightcurves of TW Hya in the energy range $0.3-2 \mathrm{keV}$ are shown in Fig. 1. The counts collected by MOS 1 and MOS 2 have been added to increase the SNR.

To investigate spectral changes during the observation we defined two energy bands, $S$ for "soft" and $H$ for "hard", and computed a hardness ratio $H R=(H-S) /(H+S)$. The overall $\mathrm{X}$-ray spectrum of TW Hya is very soft, with only a few photons recorded above $\sim 2 \mathrm{keV}$ (see discussion below). To ensure sufficient signal in both bands we chose $S=0.3-1.0 \mathrm{keV}$ and $H=1.0-2 \mathrm{keV}$. The time evolution of $H R$ is shown in the lower panel of Fig. 1. Neither the lightcurve nor the evolution of the $H R$ show conspicuous signs for flaring.

\section{High-resolution spectrum: RGS}

The RGS data of TW Hya were analysed using the standard pipeline rgsproc of the XMM-Newton Science Analysis System (SAS), version 5.4.1. We extracted the total (source plus background) and a background spectrum for TW Hya. In this paper only the first dispersion order is discussed. The full first order RGS spectrum of TW Hya is displayed in Fig. 2, except for the small range between $35-37 \AA$ where no spectral lines were found. In Fig. 2 identifications of the stronger emission lines are also provided from comparisons with line lists of the MEKAL code (Mewe et al. 1985; Mewe et al. 1995), the National Institute of Standards (NIST) data base, and the results of solar flare observations (Phillips et al. 1999). In the RGS spectrum one recognizes the lines of H-like and He-like carbon, nitrogen, oxygen and neon, while iron lines are weak or absent (especially those near 15 and $17 \AA$ ). The typical silicon and magnesium lines below $10 \AA$ are also absent in the RGS spectrum. Note, however, that at those wavelengths the RGS effective area decreases rapidly. 

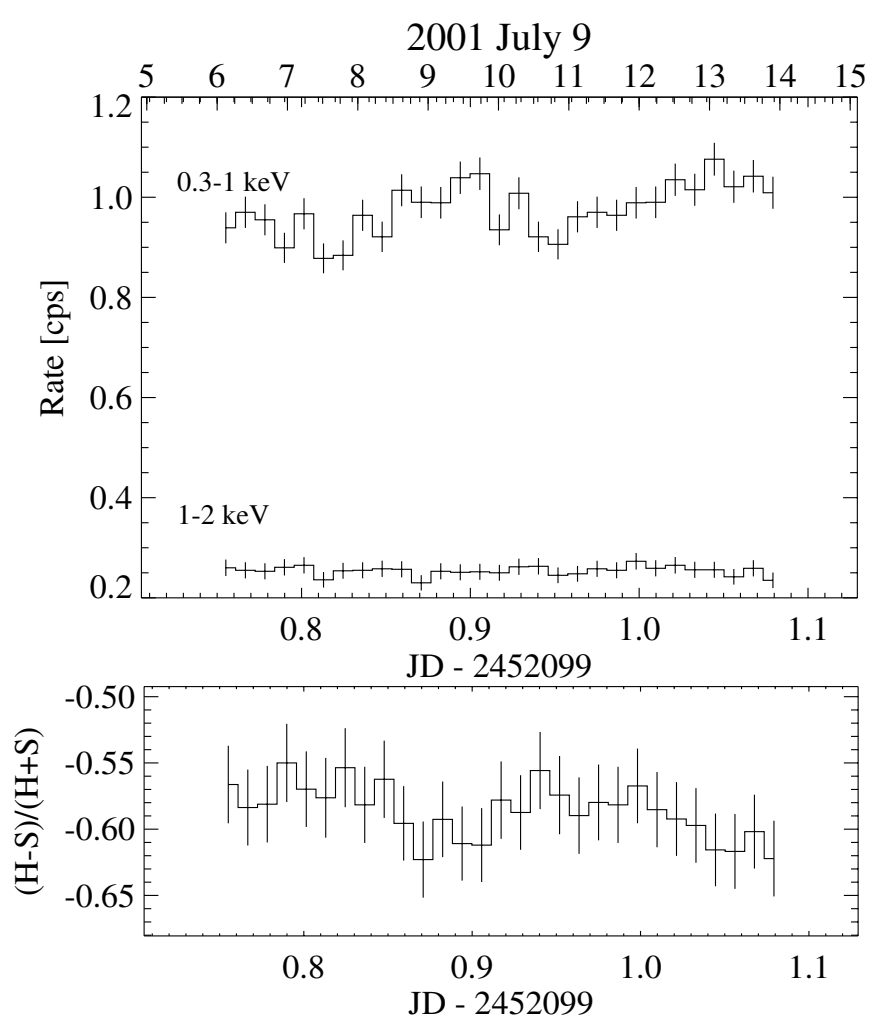

Fig. 1. Background subtracted MOS lightcurves of TW Hya in the energy range $0.3-1.0 \mathrm{keV}$ and $1.0-2.0 \mathrm{keV}$. The panel on the bottom shows the hardness ratio computed from these bands; binsize is $1 \mathrm{ks}$.

\subsection{Identification and analysis of emission lines}

To determine fluxes for individual emission lines we used the CORA $^{1}$ line fitting tool. CORA is based on a maximum likelihood technique (see Ness \& Wichmann 2002) and fits a specified number of emission lines combined with a vertical offset to account for a constant background (both instrumental and due to continuum emission from the source) assuming some specified emission line profile function. Earlier studies have shown that a Lorentzian profile provides a reasonable description of the shape of RGS line profiles (e.g. Ness et al. 2002; Stelzer et al. 2002). Before feeding the spectra into CORA we corrected for the different extraction area of total and background spectrum. To take into account possible small background variations we selected successive wavelength intervals in which the background can be approximated by a constant and performed the analysis separately for each of these intervals. Some ambiguity does remain in the determination of the local background level. To account for uncertainties associated with the background level we add a systematic error to the statistical error of the line counts. This systematic error was derived by using the highest and lowest background levels that would provide an acceptable line fit after visual inspection of the solution. Finally, the line counts and their errors (both statistical and systematic) were transformed into photon fluxes with the help of the effective areas of the RGS.

\footnotetext{
1 CORA can be downloaded from http://www.hs. uni-hamburg.de/DE/Ins/Per/Ness/Cora
}

A list of the brightest lines in the spectral range of the RGS (6-37 $\AA$ ) is provided in Table 2, where we list the lines' central wavelengths and their errors, the fitted number of counts in the line including errors and the derived photon fluxes. A suggested identification is also given. A substantial part of the total wavelength range is covered only by one instrument due to failure of one CCD on each RGS, and because of the gaps between adjacent CCDs. For lines in these regions we give the result of the active RGS, for the lines covered by both RGS we summed up the two spectra. For diagnostic purposes the most important lines are the $L y_{\alpha}$ and triplet lines of hydrogen-like and helium-like ions. The measurements of these lines for all species detected in the RGS spectrum and in addition the most prominent iron lines are provided in Table 2 . We emphasize again the unusual weakness of iron lines as one of the most remarkable features of the X-ray spectrum of TW Hya.

\subsection{Line temperatures}

For the elements neon, oxygen and nitrogen both the $L y_{\alpha}$ emission lines at $12.1 \AA, 19.0 \AA$, and $24.8 \AA$ respectively, and the He-like resonance lines at $13.5 \AA, 21.6 \AA$, and $28.8 \AA$ respectively are detected in our RGS spectra. In Fig. 3 we plot the expected energy flux ratio from the $\mathrm{H}$-like $L y_{\alpha}$ and He-like resonance lines for neon (dotted line), oxygen (solid line) and nitrogen (dashed line) versus logarithmic temperature using the CHIANTI calculations (Dere et al. 1997). We also indicate the measured line ratio range for neon (upright shading), oxygen (right tilted shading) and nitrogen (left tilted shading). These line ratios clearly correspond to effective "isothermal" temperatures, which, however, are independent of abundance. For oxygen and nitrogen these temperatures agree at $\lg T[\mathrm{~K}] \approx$ 6.45 , for neon a slightly larger effective temperature is found. The X-ray spectrum of TW Hya does not contain any strong high temperature lines despite its large overall X-ray luminosity. Thus we conclude that most of the observed line emission seems to be produced by material located at pretty much the same temperature. In fact, we verified that a 1-T thermal model (VMEKAL) with free abundances can provide an acceptable description of the RGS spectrum $\left(\chi_{\text {red }}^{2}=1.36\right.$ for 2333 d.o.f.).

However, one needs to keep in mind that the RGS effective area decreases rapidly at wavelengths below $\sim 10 \AA$, i.e. energies above $1.2 \mathrm{keV}$. Elements such as $\mathrm{Mg}$ and $\mathrm{Si}$ have their characteristic He-like and $\mathrm{H}$-like line transitions in this wavelength region. In the RGS spectrum of TW Hya these lines remain undetected because of lacking SNR, but this critical spectral region can be studied with EPIC at medium resolution (see Sect. 4). The EPIC spectrum does also show a high-energy tail that can not be explained by the rather low plasma temperatures dominating the RGS line emission (cf., Sect. 4 and Fig. 4).

\subsection{Differential emission measure}

In order to quantify a differential emission measure (DEM) distribution we adopted the following approach; see also Schmitt \& Ness (2004) for more details on this DEM reconstruction method. We first introduced a fixed temperature grid located 

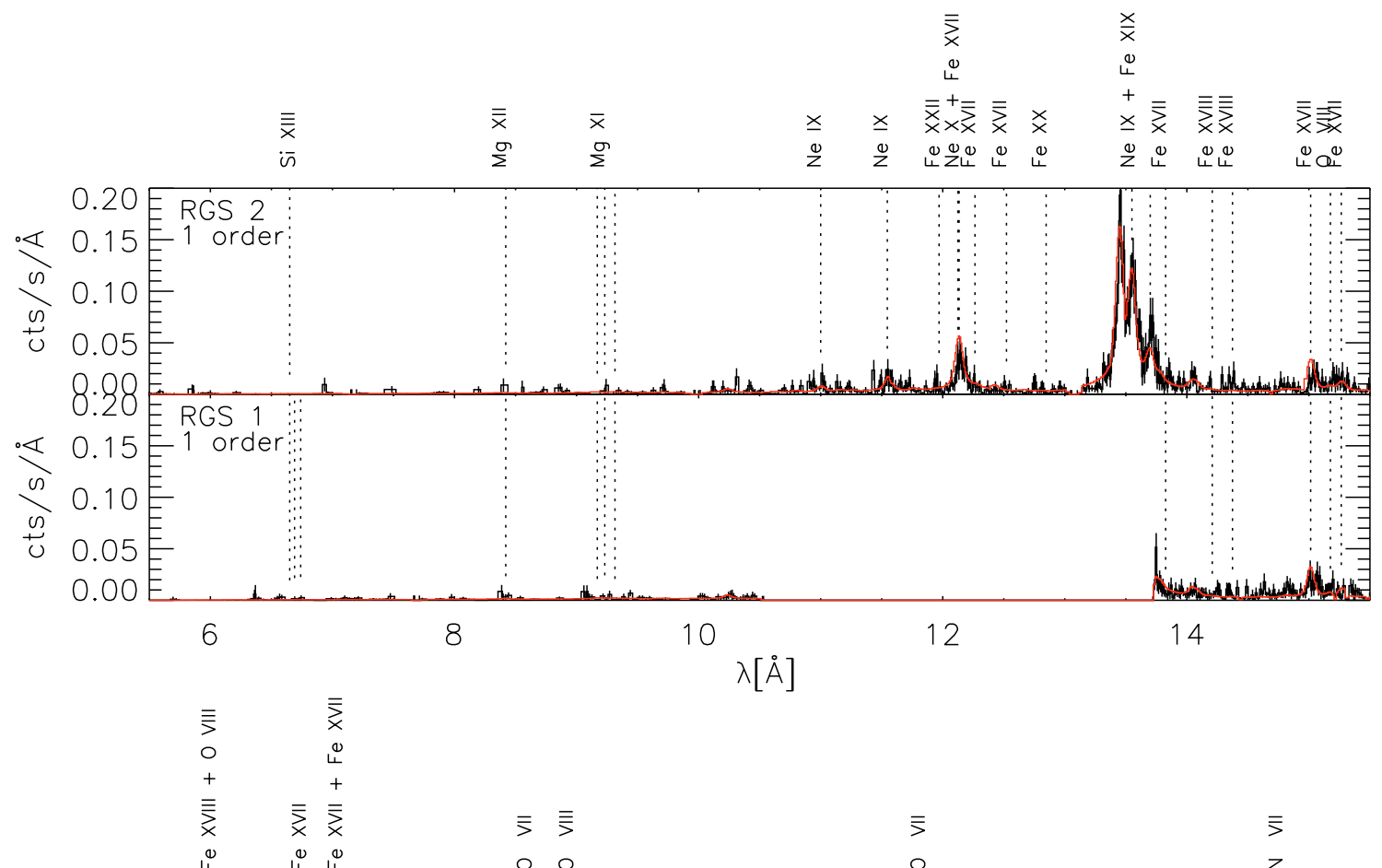

\begin{tabular}{l}
$\dot{x}$ \\
0 \\
4 \\
+ \\
$\bar{x}$ \\
$\dot{x}$ \\
\hline
\end{tabular}
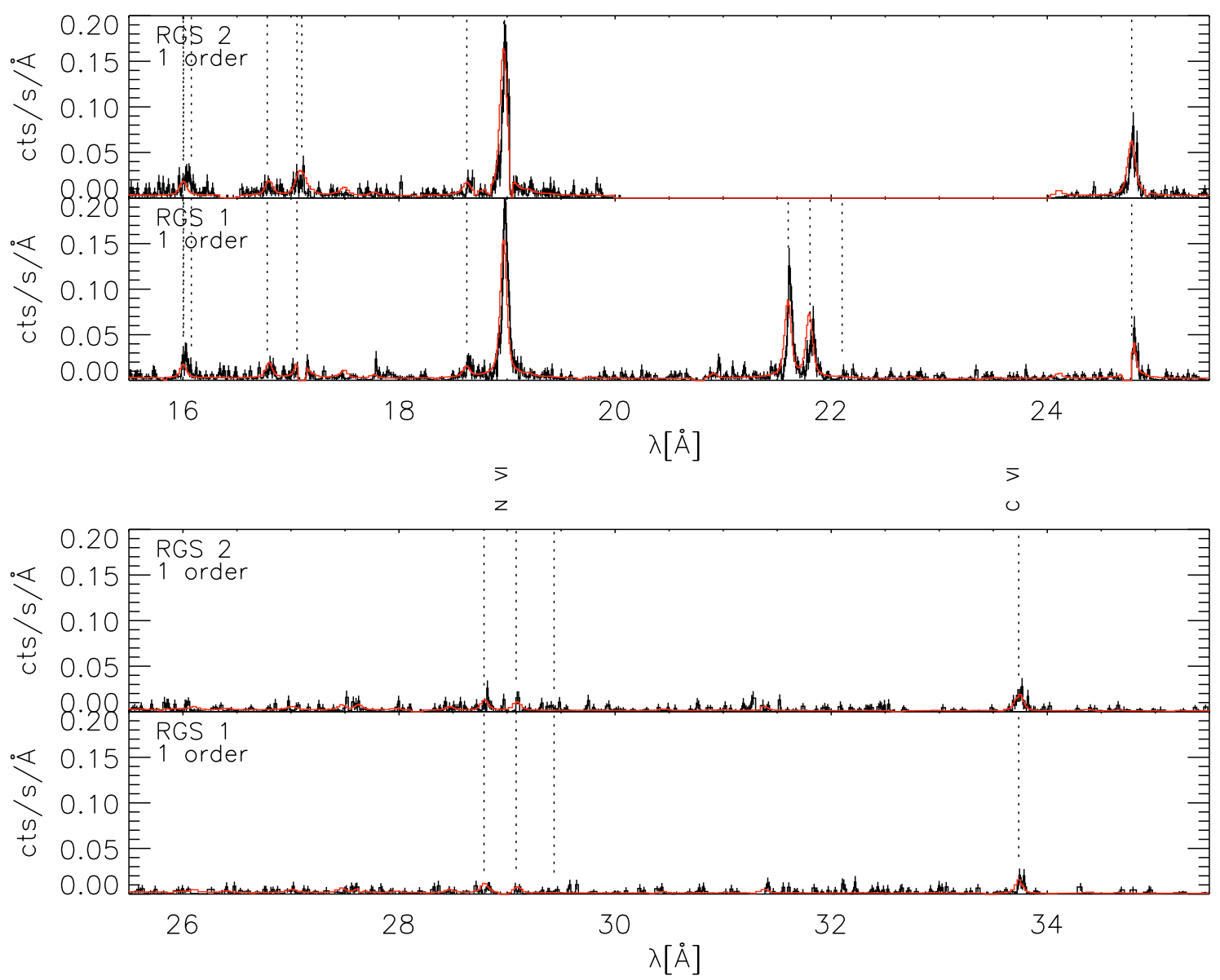

Fig. 2. First order background subtracted XMM-Newton RGS count rate spectrum of TW Hya. Overplotted is a 3-T VMEKAL model whose parameters were derived from a combination of emission line analysis and global fitting of the medium-resolution MOS spectrum. The MOS spectrum is shown together with the same model in Fig. 4, and the best fit parameters are summarized in Table 3. Exposure time is $29 \mathrm{ks}$ for each RGS. Straight horizontal lines represent gaps due to CCD chain failure or individual chip separation. Emission lines typical for stellar coronae are indicated by labels and dashed lines. 
Table 2. Spectral lines identified in the first order XMM-Newton RGS spectrum of TW Hya. left-Observed line center, width, number of counts, and photon flux. Uncertainties include statistical $1 \sigma$ error plus a systematic error stemming from the uncertainty of the local background level (see text for details). right - Line identifications from Mewe et al. (1985), Mewe et al. (1995), and Phillips et al. (1999). For line blends we list the most likely contributors according to X-ray observations of other stars.

\begin{tabular}{|c|c|c|c|c|c|c|}
\hline$\lambda$ & $\sigma$ & $I$ & Photon Flux & \multicolumn{3}{|c|}{ Identification } \\
\hline$[\AA]$ & [尺̊] & {$[\mathrm{cts}]$} & {$\left[10^{-5} \frac{\mathrm{ph}}{\mathrm{scm}^{2}}\right]$} & Ion & Trans. & $\lambda$ \\
\hline \multicolumn{7}{|c|}{ RGS 1} \\
\hline 18.983 & 0.055 & $465.7 \pm 43.7$ & $30.4 \pm 2.8$ & O VIII & $L y_{\alpha}$ & 18.97 \\
\hline 21.616 & 0.047 & $241.6 \pm 28.4$ & $17.4 \pm 2.1$ & O VII & $r$ & 21.61 \\
\hline 21.836 & 0.053 & $128.2 \pm 20.1$ & $9.5 \pm 1.5$ & O VII & $i$ & 21.80 \\
\hline 22.100 & 0.037 & $5.3 \pm 7.1$ & $0.4 \pm 0.6$ & O VII & $f$ & 22.10 \\
\hline \multicolumn{7}{|c|}{ RGS 2} \\
\hline 12.152 & 0.060 & $111.4 \pm 26.1$ & $6.3 \pm 1.5$ & $\mathrm{NeX}$ & $L y_{\alpha}$ & 12.13 \\
\hline 13.460 & 0.050 & $402.0 \pm 38.1$ & $23.1 \pm 2.2$ & Ne IX & $r$ & 13.45 \\
\hline 13.560 & 0.057 & $308.9 \pm 19.9$ & $17.4 \pm 1.1$ & $\mathrm{Ne} I X$ & $i$ & 13.55 \\
\hline 13.710 & 0.054 & $149.7 \pm 27.3$ & $8.2 \pm 1.5$ & $\mathrm{Ne} I X$ & $f$ & 13.70 \\
\hline 17.065 & 0.075 & $46.8 \pm 15.9$ & $2.7 \pm 0.9$ & Fe XVII & $N e 3 G$ & 17.06 \\
\hline 24.793 & 0.060 & $171.5 \pm 31.5$ & $11.8 \pm 2.2$ & N VII & $L y_{\alpha}$ & 24.78 \\
\hline 28.820 & 0.059 & $32.1 \pm 14.2$ & $2.9 \pm 1.3$ & N VI & $r$ & 28.79 \\
\hline 29.101 & 0.030 & $14.2 \pm 7.2$ & $1.3 \pm 0.6$ & N VI & $i$ & 29.10 \\
\hline 29.540 & 0.030 & $4.3 \pm 5.8$ & $0.4 \pm 0.5$ & $\mathrm{~N} \mathrm{VI}$ & $f$ & 29.54 \\
\hline \multicolumn{7}{|c|}{ RGS 1 + RGS 2} \\
\hline \multirow[t]{2}{*}{16.030} & 0.056 & $118.7 \pm 37.2$ & $3.5 \pm 1.1$ & Fe XVIII & & 16.00 \\
\hline & & & & O VIII & $L y_{\beta}$ & 16.01 \\
\hline 18.649 & 0.037 & $53.4 \pm 12.1$ & $1.7 \pm 0.4$ & O VII & $\mathrm{He} 3 \mathrm{~A}$ & 18.63 \\
\hline 33.757 & 0.065 & $113.9 \pm 20.7$ & $7.8 \pm 1.4$ & C VI & $L y_{\alpha}$ & 33.70 \\
\hline
\end{tabular}

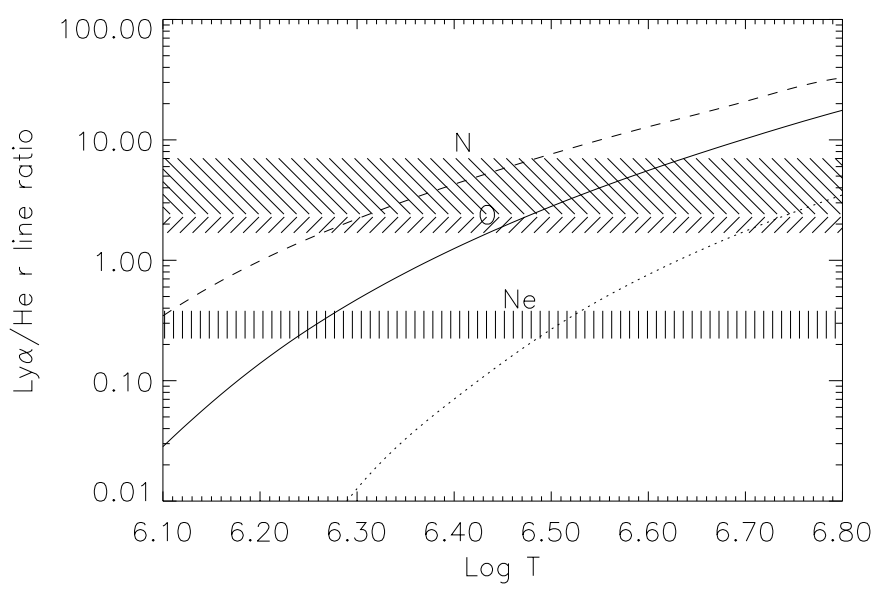

Fig. 3. Temperature dependence of the $L y_{\alpha} / r$ line ratio for neon (dotted line), oxygen (solid line), and nitrogen (dashed line) according to the CHIANTI code (Dere et al. 1997). The ratios observed in the RGS spectrum of TW Hya are indicated by the shaded patterns. All observed values are compatible with a $2-3 \mathrm{MK}$ hot isothermal plasma.

at grid points $\log T=[6.3,6.5,7.3]$. The two lower temperatures are based on the result from the above described line analysis, while the highest temperature is required for a match to the medium-resolution EPIC spectrum whose high-energy tail cannot possibly be described with a $3 \mathrm{MK}$ plasma alone. It turns out, however, that the contribution of the hot component to the overall RGS count rate is very small.

At each temperature grid point $T_{i}(i=1,3)$ we assume the presence of some emission measure $E M_{i}$. Denoting the elements carbon $(j=1)$, nitrogen $(j=2)$, oxygen $(j=3)$ and neon $(j=4)$ with the index $j$, we can then compute the line emissivities $P_{\mathrm{H}, i, j}$ and $P_{\mathrm{He}, i, j}$ for the hydrogen- and helium-like lines of the elements $j$ for the temperature components $i$. The observed line ratios $\rho_{\text {obs, } j}(j=2,4)$ can then be modelled as

$\rho_{\mathrm{mod}, j}=\frac{\sum_{i=1}^{3} E M_{i} \cdot P_{\mathrm{H}, i, j}}{\sum_{i=1}^{3} E M_{i} \cdot P_{\mathrm{He}, i, j}}$

and we seek a best fit set of the emission measures $E M_{i}$ through minimizing

$\chi^{2}=\sum_{j=2}^{4} \frac{\left(\rho_{\mathrm{obs}, j}-\rho_{\mathrm{mod}, j}\right)^{2}}{\left(\sigma_{j}\right)^{2}}$,

where $\sigma_{j}$ denotes the error of the line ratio $j$. Clearly, since we are dealing with line ratios of the same elements, the 


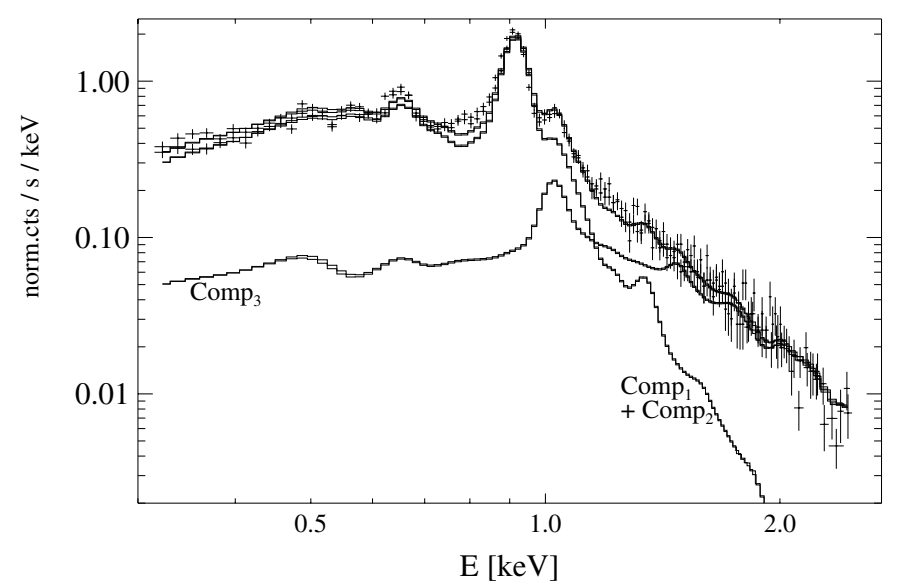

Fig. 4. EPIC MOS 1 and MOS 2 spectrum of TWHya. The data is overlaid by the best fit 3-T VMEKAL model B from Table 3; see also Fig. 2 where the same model is folded with the RGS response. The individual contributions of the two lower-temperature components and of the high-temperature component are also shown.

normalization can be set arbitrarily (we chose $E M_{1}=1$ ). The thus derived $E M$-distribution is abundance independent. The best fit values for the (relative) values of $E M_{\mathrm{i}}$ are found to be $[1.0,2.35,0.59]$. This choice of emission measure reproduces the observed line ratios extremely well $\left(\chi_{\text {red }}^{2} \approx 0.5\right)$. However, given the ill-conditioned nature of the reconstruction problem, other choices of the EM-distribution also provide statistically acceptable fits. With the thus derived set of best fit $E M_{i}$ values we can choose the normalization to reproduce, say, the oxygen $L y_{\alpha}$ line fluxes. Then we use this normalization to determine abundances of carbon, nitrogen, neon and iron relative to oxygen by forcing agreement between predicted and measured fluxes in the observed lines. Note that for carbon and iron we can only use the $L y_{\alpha}$ line (for carbon) and the Fe XVII 17.07 Å line (for iron). Using an interstellar hydrogen column density of $N_{\mathrm{H}}=2 \times 10^{20} \mathrm{~cm}^{-2}-$ which is required for a good fit of the EPIC data and is in agreement with the expected interstellar absorption towards TW Hya - we find with this procedure the values $\mathrm{C} / \mathrm{O}=1.07, \mathrm{~N} / \mathrm{O}=3.15, \mathrm{Ne} / \mathrm{O}=9.77$ and $\mathrm{Fe} / \mathrm{O}=0.91$ with respect to cosmic abundances. Realistic abundance errors are difficult to determine. We estimate abundance errors of about $20 \%$ for neon, and about $30 \%$ for carbon, nitrogen and iron.

\section{EPIC medium resolution spectrum: EPIC}

The count rate spectrum of each EPIC MOS detector was extracted from the area described in Sect. 2. We extracted and analysed the EPIC spectra with SAS version 5.4.1. Individual redistribution matrices and an ancilliary response file were generated in the course of our data reduction process. The combination of these two files fully models the instrumental response.

The EPIC spectrum has a pronounced peak at $E \sim 0.91 \mathrm{keV}$ or $13.6 \AA$. Since we know from the XMM-Newton RGS spectrum that both the continuum and iron line emissions are weak, we conclude that this spectral bump must represent emission from $\mathrm{H}$ - and He-like neon. This remarkable feature is not
Table 3. Best fit parameters for the X-ray spectrum of TW Hya described by a multi-temperature thermal model (VMEKAL) including photo-absorption $\left(N_{\mathrm{H}}=2 \times 10^{20} \mathrm{~cm}^{-2}\right)$. The electron density was fixed to $5 \times 10^{12} \mathrm{~cm}^{-3}$. Elements not listed below are irrelevant in this spectrum, and their individual abundances were held fixed at the solar value. The temperatures and the coupling between some parameters were pre-determined by the analysis of the emission lines seen in the RGS spectrum. Values for the abundances are given with respect to the solar photosphere, $\log T$ is in $\mathrm{K}$, and $\log E M$ in $\mathrm{cm}^{-3}$. (A) - Best fit 3-T model to the EPIC MOS spectrum in the range $\lambda=5-40 \AA$. (B) - Same as (A) with free $\mathrm{Mg}$ and $\mathrm{Si}$ abundances.

\begin{tabular}{lrrrr}
\hline & \multicolumn{2}{c}{ Model A } & & \multicolumn{2}{c}{ Model B- } \\
\cline { 2 - 3 } \cline { 5 - 5 } Parameter & Param. & Param. & Param. & P aram. \\
\hline $\mathrm{C}$ & $=1.07 \times \mathrm{O}$ & $=0.28$ & $=1.07 \times \mathrm{O}$ & 0.40 \\
$\mathrm{~N}$ & $=3.14 \times \mathrm{O}$ & $=0.82$ & $=3.14 \times \mathrm{O}$ & 1.18 \\
$\mathrm{O}$ & & 0.26 & & 0.37 \\
$\mathrm{Ne}$ & & 3.15 & & 3.97 \\
$\mathrm{~S}$ & & $=0.0$ & & $=0.0$ \\
$\mathrm{Mg}$ & & $=1.0$ & & 0.65 \\
$\mathrm{Si}$ & & $=1.0$ & & 0.13 \\
$\mathrm{Fe}$ & & 0.26 & & 0.24 \\
\hline $\log T_{1}$ & & $=6.3$ & & $=6.3$ \\
$\log T_{2}$ & & $=6.5$ & & $=6.5$ \\
$\log T_{3}$ & & $=7.3$ & & $=7.3$ \\
\hline $\log E M_{1}$ & & 52.64 & & 52.53 \\
$\log E M_{2}$ & $2.35 \times E M_{1}$ & $=53.01$ & $2.35 \times E M_{1}$ & $=52.90$ \\
$\log E M_{3}$ & $0.59 \times E M_{1}$ & $=52.41$ & & 52.53 \\
\hline$\chi_{\text {red }}^{2}($ d.o.f. $)$ & $2.79(233)$ & & $2.61(232)$ \\
\hline
\end{tabular}

observed in EPIC data of other young stars (see e.g. Güdel et al. 2001; Stelzer et al. 2002; Stelzer \& Neuhäuser 2001b), emphasizing the peculiar nature of the X-ray spectrum of TW Hya.

Approximating the MOS spectra by a sum of three thermal models with fixed values of temperature and based on the parameters derived from the line analysis, we verify that the temperature and abundance structure derived from the analysis of individual emission lines also provides a consistent description of the medium-resolution data. In this global fit to the MOS spectrum an absorption term with atomic cross-sections of Morrison \& McCammon (1983) and $N_{\mathrm{H}}=2 \times 10^{20} \mathrm{~cm}^{-2}$ is applied to describe the effects of inter- and/or circumstellar cold gas. The electron density was set to $5 \times 10^{12} \mathrm{~cm}^{-3}$ following the result of our line-based density analysis (see Sect. 5.2) and kept fixed. We restricted our modeling to the 5.5-40 range, and used the VMEKAL code as implemented in XSPEC, version 11.2.0.

We first allowed only the oxygen abundance and the absolute value of the emission measure to vary, i.e., the parameters not constrained by our line modeling. The best fit determined in this fashion results in $\chi_{\text {red }}^{2}=3.2$ (235 d.o.f.), and the residuals suggest that a slight adjustment of the abundances may be needed. If we allow the neon and iron abundances 


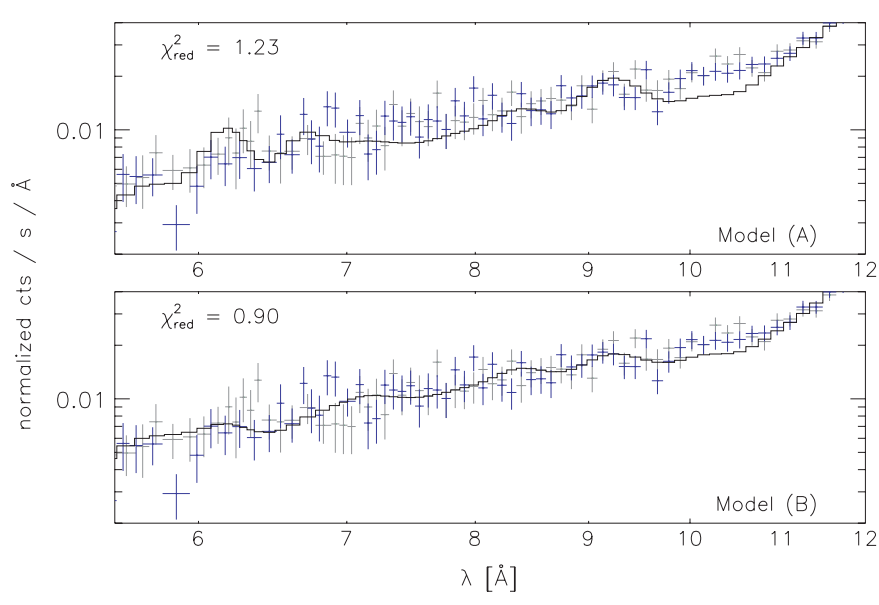

Fig. 5. Close-up of the EPIC MOS 1 and MOS 2 spectrum of TW Hya in the range 5.5-12 ̊: top - Model (A) from Table 3: Mg and Si abundance fixed to solar; bottom - Model (B) from Table 3: Mg and $\mathrm{Si}$ abundance free parameters resulting in subsolar abundances for both elements.

to de-couple from the oxygen abundance, the fit improves to $\chi_{\text {red }}^{2}=2.8$ (233 d.o.f.). Note that these "new" abundances are still within the errors of the abundances derived from our linebased RGS analysis. The best fit parameters of this model are summarized in Table 3 (Model A). We point out that we do not derive nitrogen and carbon abundances from the EPIC spectra, because the instrument has little sensitivity in the relevant spectral range. These abundances are solely derived from the RGS data and - for carbon - are based only on one single line.

Up to this point our fit results are in good agreement with the findings presented by Kastner et al. (2002) from their analysis of the Chandra HETG spectrum of TW Hya, i.e., a strong over-abundance of $\mathrm{Ne}$ and emission dominated by lowtemperature plasma. Inspection of the EPIC fit residuals does however show clear discrepancies between the EPIC data and model at $\lambda<14 \AA$, where the H-like $L y_{\alpha}$ and He-like triplet transitions of magnesium and silicon are located. In particular, two bumps in the model come from emission of Si XIV and $\mathrm{Mg}$ XII without any counterpart in the data. Therefore we allowed for adjustment of the $\mathrm{Mg}$ and $\mathrm{Si}$ abundances in the spectral fitting process. Since any changes in the $\mathrm{Mg}$ and $\mathrm{Si}$ abundances will be compensated for by changes in $E M_{3}$, we must also let vary $E M_{3}$. The resulting best fit models have magnesium and silicon abundances well below solar values with a best-fit $\chi_{\text {red }}^{2}$ of 2.61 . The $90 \%$ error contour of the magnesium is at 0.94 (w.r.t. solar), and the silicon abundance is definitely subsolar. A summary of all parameters of this model is given in Table 3 (Model B). In Fig. 4 we show the best fit 3-T model together with the MOS data. For illustration the sum of the two low-energy components and the high-energy component are also plotted separately.

In order to point out the effects of changing $\mathrm{Si}$ and $\mathrm{Mg}$ abundances we consider only the range between 5.5-12. . In Fig. 5 we confront the two models from Table 3 in this wavelength interval with each other. Clearly the fit with free abundances describes the EPIC data far better than assuming solar abundances for silicon and magnesium. In the 5.5-12 interval the $\chi_{\text {red }}^{2}$ decreases from 1.23 (102 d.o.f.) for the model with solar $\mathrm{Mg}$ and $\mathrm{Si}$ to 0.90 (101 d.o.f.) for the model where $\mathrm{Mg}$ and $\mathrm{Si}$ are free parameters, leading to subsolar abundances for both elements.

As a final consistency check of our procedure we now folded back the best fit model B with the response of the RGS. The result is overplotted on the RGS data in Fig. 2. As expected, we find good agreement between model and data. The emission measure of the high- $T$ component is about a factor of three lower than the sum of the two low- $T$ components. This together with its high temperature leads to a very low contribution to the RGS count rate of $0.015 \mathrm{cps}$ (RGS 1 ) and $0.021 \mathrm{cps}$ (RGS 2), implying that the high- $T$ component is essentially undetectable in the RGS. (The lower count rate in RGS 1 is caused by the missing CCD in the 10-14 $\AA$ interval.) We thus conclude that the model $\mathrm{B}$ described in Table 3 provides an acceptable description of both the XMM-Newton EPIC and RGS spectra.

\section{Discussion}

So far TW Hya is the only cTTS, i.e. a PMS star with strong $\mathrm{H}_{\alpha}$ emission, for which a high-resolution XMM-Newton X-ray spectrum is available. Since in young stars $\mathrm{H}_{\alpha}$ emission is related to accretion TW Hya presents a unique test case for X-ray emission in this early phase of stellar evolution. Furthermore, it is one of the few stars observed with both XMM-Newton and Chandra at high spectral resolution. In the following we discuss the properties of the X-ray emission of TW Hya, and we present a physical scenario explaining it.

\subsection{Variability}

During the Chandra observation of TW Hya discussed by Kastner et al. (2002) a sharp rise in intensity followed by a nearly linear decay occurred, that the authors interpreted as a flare. We find little evidence for variability in the XMM-Newton observation presented here (acquired $\sim 1$ yr after the Chandra data). In any case the lightcurve does not carry the clear signatures of a flare, and no change in spectral hardness is observed. Similarly Kastner et al. (2002) found no changes in the spectral parameters during the variable phase of the Chandra observation, very untypical for stellar (flare) activity. The flare-like event in the Chandra observation has a decay time of $\sim 10 \mathrm{ks}$. Assuming a flare loop at $T \sim 3 \mathrm{MK}$ (the dominating temperature derived from the X-ray spectrum) that decays through radiative cooling the density should be $\sim 10^{10} \mathrm{~cm}^{-3}$. This is in sharp contrast to the result from the analysis of line ratios (see Sect. 5.2), according to which the densities are higher by two orders of magnitude.

We directly compare the emission levels by computing X-ray fluxes for the time-averaged spectrum. From the Chandra HETGS spectrum Kastner et al. (2002) derived an $\mathrm{X}$-ray flux of $2.5 \times 10^{-3}$ photons $\mathrm{cm}^{-2} \mathrm{~s}^{-1}$ for the $0.45-6.0 \mathrm{keV}$ band. Integrating the RGS spectrum in the energy band which comes nearest to the spectral range of the HETGS, i.e. 5.5-27.5 $\AA$ or $0.45-2.25 \mathrm{keV}$, we arrive at a similar value of $f_{\mathrm{x}, \mathrm{RGS} 1} \approx f_{\mathrm{x}, \mathrm{RGS} 2}=2.3 \times 10^{-3}$ photons $\mathrm{cm}^{-2} \mathrm{~s}^{-1}$ corresponding to $L_{\mathrm{x}, \mathrm{RGS}}=1.4 \times 10^{30} \mathrm{erg} / \mathrm{s}$ at a distance of $57 \mathrm{pc}$. 


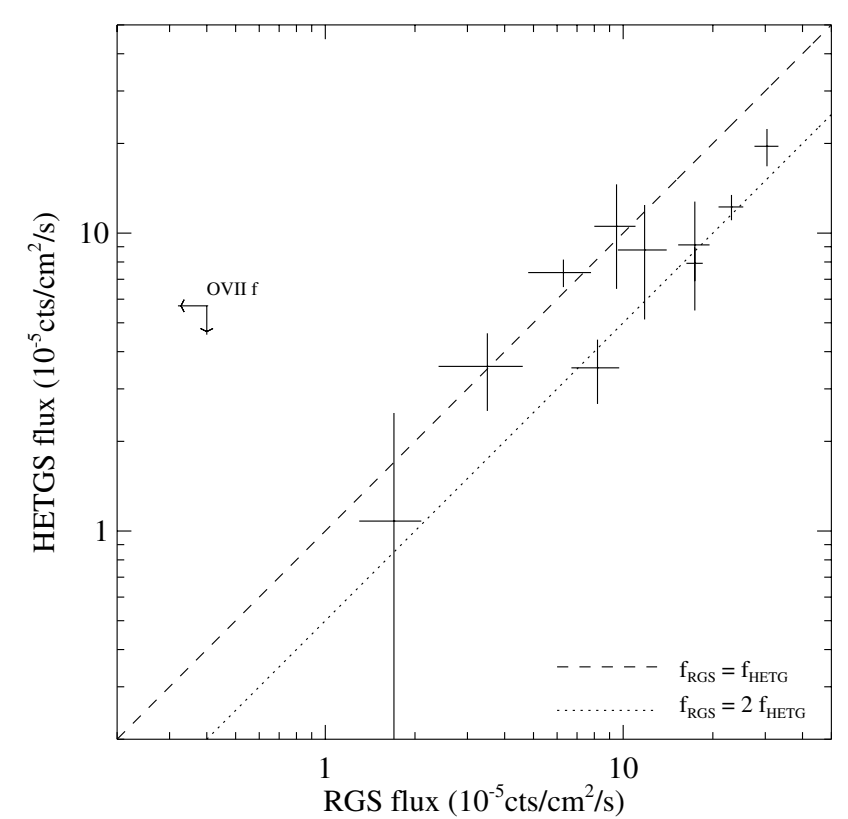

Fig. 6. Comparison of photon fluxes for individual emission lines in the X-ray spectrum of TW Hya measured with the XMM-Newton RGS (see Table 2) and the Chandra HETGS-MEG (see Kastner et al. 2002). The XMM-Newton and Chandra observations are separated by about one year. All lines are constant within about a factor of two.

This suggests that TW Hya exhibits only moderate long-term variability.

Based on the small amplitude of long-term variations discussed above, comparing fluxes measured for individual emission lines allows to check the calibration of the instruments on both satellites. We observe a trend towards slightly higher fluxes in the RGS as compared to the HETGS (Fig. 6). However, the difference is a factor two at most.

\subsection{Density in the $X$-ray emitting region}

The high-resolution X-ray spectrum obtained with the RGS allows us to measure flux ratios between individual emission lines and use them for plasma diagnostics. The triplets of He-like ions are of particular importance, because ratios between the intensity of resonance $r$, intercombination $i$, and forbidden $f$ line are sensitive to (electron) temperature (through $G=(f+i) / r$ ) and density (through $R=f / i)$. While the $G$-ratio is of limited value for temperature diagnostics, the $R$-ratio constitutes a unique tool to probe the electron density in stellar coronae. Although the triplets from silicon (Si XIII) to nitrogen (N VI) fall in the spectral range of the RGS, in TW Hya only three elements - nitrogen, oxygen, and neon - show sufficient emission in the respective transitions of their He-like ions to warrant analysis.

In Fig. 7 we zoom in on the spectral region around the oxygen and neon triplets of TW Hya overlaying the data with our line fit result. For the modeling of both triplets we used three Lorentzians representing the $r, i$, and $f$ lines. Some caution is in order when interpreting the spectrum near the position of the Ne IX triplet: the triplet is not fully resolved in the RGS, and is known to form a blend with a number of iron lines, predominantly a Fe XIX line at $13.53 \AA$ that contaminates the $\mathrm{Ne}$ intercombination line. However, we performed fits of the neon triplet including iron and find undistinguishable results. Since we find little hints for the presence of Fe XIX (and generally any iron) in the RGS spectrum (e.g. the lines at $13.80 \AA$ and $14.66 \AA$ are not seen) we assume that iron contamination plays only a minor role and can be neglected.

The remarkable feature of the He-like triplets of TW Hya is the weakness of the forbidden lines. In the LETGS and RGS spectra of all late-type stars observed so far the forbidden lines are always stronger than the intercombination line (in oxygen and neon) and in many cases the measurements are consistent with the so-called low-density limit (e.g. Ness et al. 2001, 2002; Stelzer et al. 2002). This is definitely not the case for TW Hya. We note in particular that with TW Hya being rather cool (spectral type K7; Rucinski \& Krautter 1983) the low intensity of the forbidden line cannot be attributed to UV radiation at the wavelengths corresponding to the $f \longrightarrow i$ transitions (Costa et al. 2000). Instead it is a direct indicator of high densities. The formally derived $f / i$-value for O VII is $R_{\mathrm{O} \text { VII }}<$ 0.16 , i.e., only an upper limit can be given due to the weakness of the O VII $f$-line. Comparing our measurements to recent calculations by Porquet et al. (2001) without any radiation field we find a lower limit on the density of $n_{\mathrm{e}, \mathrm{O} \text { VII }}>1 \times 10^{12} \mathrm{~cm}^{-3}$. This is corroborated by the low flux of the nitrogen forbidden line which is only marginally detected (cf. Table 2 ). The $R$-ratio for neon $\left(R_{\mathrm{NeIX}}=0.47 \pm 0.11\right)$ indicates a density of $n_{\mathrm{e}, \mathrm{Ne}} \mathrm{IX} \sim 1 \times 10^{13} \mathrm{~cm}^{-3}$. Inclusion of the Fe XIX blend in the fit of the neon triplet would lower the strength of the $i$ line, and lead to a somewhat higher value for $R_{\mathrm{Ne} \text { IX }}(0.62 \pm 0.12)$, and subsequently lower density. However the numbers are compatible with the ones cited above within the statistical uncertainties.

The densities derived from the oxygen and neon triplets are at least two orders of magnitude above typical coronal densities. Our density measurements thus fully confirm the results derived by Kastner et al. (2002) from their HETGS spectrum, and we conclude in particular that the high densities cannot be a result of the flaring reported by Kastner et al. (2002) in their Chandra data.

\subsection{Elemental abundances}

The XMM-Newton RGS spectrum as well as our spectral fits to the EPIC spectrum show the X-ray emission of TW Hya clearly dominated by neon emission in agreement with Kastner et al. (2002). Our results specifically suggest a neon abundance significantly enhanced with respect to the solar value $(\mathrm{Ne} \sim$ $4 \mathrm{Ne}_{\odot}$ ), while iron and oxygen, the elements with the largest number of lines in the sensitive region of the XMM-Newton and Chandra instruments, are underabundant with respect to solar values, again in agreement with Kastner et al. (2002). For the elements silicon, magnesium, and nitrogen Kastner et al. (2002) claim solar abundances, while carbon lines were not covered by the HETGS spectrum. Interestingly, the lines of He-like and H-like silicon are clearly visible in the HETGS spectrum of TW Hya (cf., Fig. 2 in Kastner et al. 2002), while the same authors note "a curious lack of $\mathrm{Mg}$ features, relative 

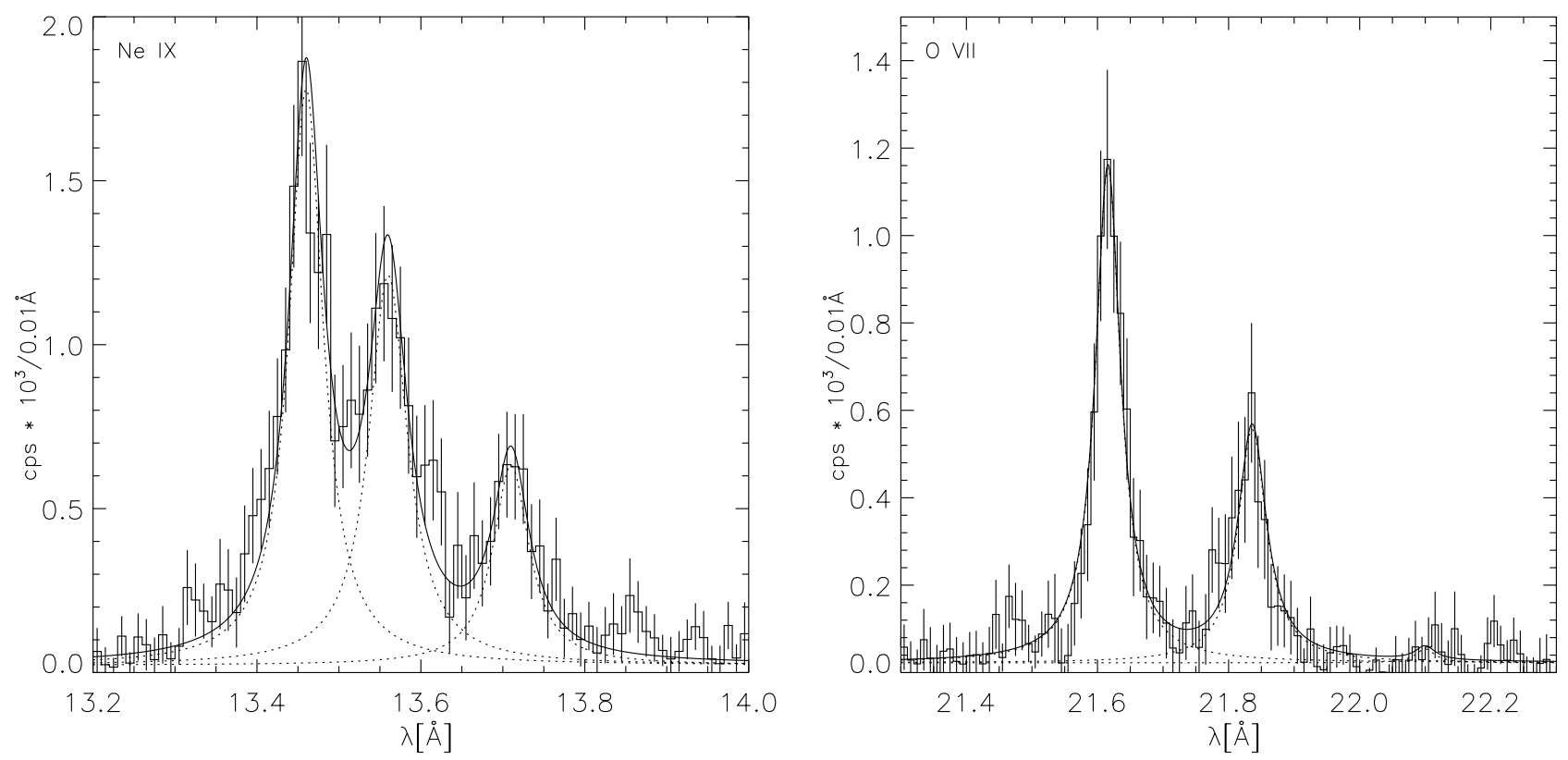

Fig. 7. He-like triplets of Ne IX and O VII measured with the RGS together with best fit Lorentzians. The continuum close to each triplet is approximated by a straight horizontal line. The intensity enhancement to the right of the Ne IX triplet in the RGS spectrum is due to Fe XVII. But the major contamination of the Ne IX triplet is expected to come from a Fe XIX line which is unresolved from the triplet intercombination line (see text in Sect. 5.2).

to the [i.e. their] model". While the RGS spectra are not sufficiently sensitive in the relevant spectral region, the EPIC spectrum clearly suggests subsolar abundances also for magnesium and silicon.

Let us now focus on the line emission from neon, oxygen, nitrogen, and carbon as seen by the RGS. Our spectral analysis both with RGS and EPIC shows that very little low temperature material (with $\log T[\mathrm{~K}]<6.3$ ) can be present. Ness et al. (2002) report photon flux ratios for $L y_{\alpha} / r$ of 0.59 (oxygen) and 0.94 (nitrogen) for the low-temperature F-star Procyon, and 1.87 (oxygen) and 3.26 (nitrogen) for the highertemperature K-star $\epsilon$ Eri, which must be compared to the values of 1.75 (oxygen) and 4.07 (nitrogen) measured for TW Hya despite its overall low temperature. Although DEM distributions are typically not unique because of the ill-defined nature of the inversion problem, we want to emphasize that the peculiar chemical abundances of TW Hya are not caused by our choice of the $E M$ distribution. For this we examined the energy flux ratio between the $L y_{\alpha}$ lines of C VI and N VII as a function of temperature and find that this line ratio can not be explained by any single temperature or combination of temperatures under the assumption of solar abundances for both elements (Fig. 8a). Therefore nitrogen must be overabundant relative to carbon (and also oxygen) with respect to the solar abundance ratio, again in accordance with our fit results. We then investigated the flux ratio between the $r$ line of neon and the oxygen $L y_{\alpha}$ line in the same way (Fig. 8b), and again the observed flux ratio can not be reproduced by any single temperature or combination of temperatures if the abundances were solar, implying that neon must be overabundant compared to oxygen with respect to solar abundances, as found in our fit results. Finally, inspecting the flux ratio between the C VI $L y_{\alpha}$ line and the O VII $r$ line we find perfect consistency assuming the $\mathrm{C} / \mathrm{O}$ ratio being solar (Fig. 8c). Since our EPIC results suggest that silicon, magnesium, iron, and oxygen all have similar abundance (with the possible exception of magnesium) we thus conclude that the elemental abundances of these elements as well as that of carbon are reduced with respect to solar values by about a factor of 3-4. Nitrogen is enhanced with respect to these elements by a factor of 3 as evidenced by the XMM-Newton RGS spectrum, and neon is enhanced by a factor of 10 as evidenced by both the XMM-Newton RGS and EPIC spectra.

\subsection{Interpretation}

The three properties distinguishing the X-ray spectrum of TW Hya from that of "normal" late-type stars are (1) the absence of a hot component with large emission measure, (2) the high density of the X-ray emitting region, and (3) the peculiar elemental composition, and in particular the low abundance of iron. The first two properties can be naturally explained by attributing the X-ray emission to an accretion shock, the third by the specific environment of TW Hya.

Employing the strong shock formula

$$
T=\frac{3 \mu m_{\mathrm{H}} V_{\mathrm{pre}}^{2}}{16 k}
$$

with $\mu$ denoting the mean molecular weight, $m_{\mathrm{H}}$ the hydrogen atom mass, and $k$ Boltzmann's constant, we can convert the measured temperature $\lg T[\mathrm{~K}]=6.45$ to an infall preshock velocity $V_{\text {pre }}$ of about $350 / \sqrt{(\mu)} \mathrm{km} \mathrm{s}^{-1}$. The preshock particle density $n_{\text {pre }}$ should be one fourth of the measured post shock density $n_{\text {post }}$, i.e., $2.5 \times 10^{12} \mathrm{~cm}^{-3}$. In order to estimate $L_{\text {post }}$, the thickness of the postshock cooling layer, we 

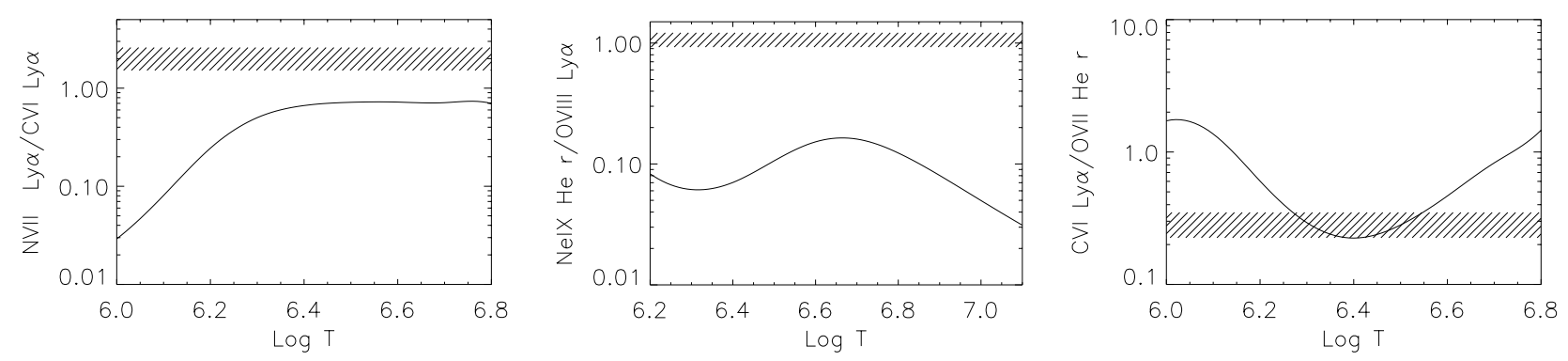

Fig. 8. Temperature dependence of various line ratios measured in the RGS spectrum of TW Hya. Solid lines are CHIANTI model calculations (Dere et al. 1997), shaded regions denote the $1 \sigma$ range observed with the RGS. a) $-\mathrm{N}$ VII $L y_{\alpha} / \mathrm{C}$ VI $L y_{\alpha} ;$ b) $-\mathrm{Ne}$ IX $r / \mathrm{O}$ VIII $L y_{\alpha}$; c) - C VI $L y_{\alpha} / \mathrm{O}$ VII $r$. The plots demonstrate that the $\mathrm{C} / \mathrm{O}$ abundance ratio is close to solar, while the $\mathrm{N} / \mathrm{O}$ and Ne/O abundance ratio can not possibly be solar.

equate the infalling kinetic energy flux with the radiated energy flux (Lamzin 1995) through

$$
\frac{\mu m_{\mathrm{H}} n_{\text {pre }} V_{\text {pre }}^{3}}{2}=n_{\text {post }}^{2} \Lambda_{\text {mean }} L_{\text {post }}
$$

Here $\Lambda_{\text {mean }}$ denotes the total mean radiative post shock cooling rate per unit emission measure. Denoting $\Lambda_{\text {mean }}$ in units of $10^{-23} \mathrm{erg} \mathrm{cm}^{3} \mathrm{~s}^{-1}$, we derive

$L_{\text {post }}=\frac{9 \times 10^{7}}{\sqrt{\mu}}\left(\Lambda_{\text {mean }, 23}\right)^{-1}[\mathrm{~cm}]$.

For an isothermal cosmic abundance plasma at $\lg T[\mathrm{~K}]=6.45$ $\Lambda_{\text {mean,23 }}$ is generally close to unity; if we assume $\Lambda_{\text {mean,23 }}=0.3$ because of the depleted elemental abundances and $\mu=1.5$ the post-shock thickness is $L_{\text {post }} \approx 2500 \mathrm{~km}$ with admittedly considerable uncertainty. Using the observed O VIII energy flux of $3.2 \times 10^{-13} \mathrm{erg} / \mathrm{cm}^{2} / \mathrm{s}$ and an assumed O VIII $L y_{\alpha}$ cooling function of $9.6 \times 10^{-25} \mathrm{erg} \mathrm{cm}^{3} \mathrm{~s}^{-1}$ (assuming $\lg T[\mathrm{~K}]=6.45$ and an oxygen depletion of 0.3 ) we compute an overall volume emission measure of $E M=1.3 \times 10^{53} \mathrm{~cm}^{-3}$ at the distance of $57 \mathrm{pc}$. Since $n_{\text {post }} \approx 10^{13} \mathrm{~cm}^{-3}$, the emitting volume $V_{\text {emit }}$ must be $V_{\text {emit }}=1.3 \times 10^{27} \mathrm{~cm}^{3}$. The shock area $A_{\text {shock }}$ then becomes $A_{\text {shock }}=V_{\text {emit }} / L_{\text {post }}=5.1 \times 10^{18} \mathrm{~cm}^{2}$, which would constitute only a very tiny fraction of the visible stellar surface $(<0.01 \%)$. Clearly, these estimates are by necessity very rough, but in qualitative agreement with previous observations in other wavelengths: Muzerolle et al. (2002) have shown that the shape of the optical/UV spectral energy distribution of TW Hya can be explained by an accretion shock which fills $\sim 0.3 \%$ of the stellar surface, and small accretion shocks are also found on other T Tauri stars (Calvet \& Gullbring 1998). Computing the mass accretion rate in the funnel flow through $M_{\text {acc }}=1 / 4 A_{\text {shock }} n_{\text {post }} \mu m_{H} V_{\text {pre }}$ we find $M_{\text {acc }} \sim 1 \times 10^{-11} M_{\odot} / \mathrm{yrs}$, which is entirely plausible and within one order of magnitude of the mass accretion rate of TW Hya derived from UV data (Muzerolle et al. 2002).

What kind of material is flowing through this funnel onto TW Hya? We have shown that this material is in general metal-depleted in particular with respect to grain-forming elements such as $\mathrm{Mg}, \mathrm{Si}, \mathrm{Fe}, \mathrm{C}$ and $\mathrm{O}$. Overabundances of neon are found in stellar coronae of RS CVn systems (Audard et al. 2003), and nitrogen overabundances in evolved stars (Schmitt \& Ness 2002). The low abundances especially of iron and carbon call for a different explanation for these chemical peculiarities. TW Hya is rather young and formed "just recently" out of a molecular cloud. Models of cloud chemistry at high densities $\left(n>10^{7} \mathrm{~cm}^{-3}\right)$ predict that almost all metals condense into grains except nitrogen (Charnley 1997). If the remaining depleted gas is then decoupled from the grains in an accretion disk around a young star (as is expected in planetary formation models), the accreted and shock-heated gas is expected to be metal-deficient as well. Indeed, Weinberger et al. (2002) found evidence for the presence for condensed silicates in the disk of TW Hya, and Herczeg et al. (2002) speculated about a deficiency of $\mathrm{Si}$ in the accretion flow of TW Hya based on the examination of its far-UV spectrum. XMM-Newton has completed this picture of TW Hya as an accreting PMS star, and for the first time provided a consistent view of X-ray emission from a stellar accretion shock by observation.

\section{Summary}

To summarize, the marked differences between the X-ray properties of TW Hya and active main-sequence dwarf stars suggest either (i) strong evolutionary effects on coronal structures, or (ii) an entirely different origin for the X-ray emission of TW Hya. X-ray emission from a shock at the bottom of an accretion column provides a plausible mechanism for the latter possibility. The derived shock temperatures, gas densities, filling factors and mass accretion rates as well as the obvious lack of grain-forming elements in the $\mathrm{X}$-ray emitting region provide strong indications in favor of this scenario.

A high-resolution X-ray spectrum of another member of the TW Hya association, HD 98800, was recently obtained with Chandra ([arXiv: astro-ph/0403062]). The object is a quadruple star with a circumbinary disk. In terms of $\mathrm{H}_{\alpha}$ emission HD 98800 is a wTTS. Therefore it does not come as a surprise that its X-ray properties are very different from those of TW Hya, and clearly reminiscent for typical stellar activity. Similarly, the high-resolution X-ray observation of PZ Tel, a 20 Myr old post-TTS in the Tucanae association, does not show properties drammatically different from older late-type stars (Argiroffi et al. 2004).

We conclude that TW Hya stands out as a presently unique example for a star with strong evidence for accretion signatures in its X-ray spectrum. Future high spectral resolution and 
high sensitivity X-ray observations will have to show whether soft emission, high densities, and metal-deficiency are general characteristics of cTTS.

Acknowledgements. BS acknowledges financial support from the European Union by the Marie Curie Fellowship Contract No. HPMDCT-2000-00013. X-ray astronomy at the Hamburger Sternwarte is generously supported by DLR. We have used the CORA line fitting package kindly provided by J.-U. Ness. This work is based on observations obtained with $X M M$-Newton, an ESA science mission with instruments and contributions directly funded by ESA Member States and the USA (NASA).

\section{References}

Alcalá, J. M., Krautter, J., Schmitt, J. H. M. M., et al. 1995, A\&AS, 114,109

Argiroffi, C., Drake, J. J., Maggio, A., et al. 2004, ApJ, in press

Audard, M., Güdel, M., Sres, A., Raassen, A. J. J., \& Mewe, R. 2003, A\&A, 398, 1137

Calvet, N., \& Gullbring, E. 1998, ApJ, 509, 802

Charnley, S. B. 1997, MNRAS, 291, 455

Costa, V. M., Lago, M. T. V. T., Norci, L., \& Meurs, E. J. A. 2000, A\&A, 354, 621

Dere, K. P., Landi, E., Mason, H. E., Monsignori Fossi, B. C., \& Young, P. R. 1997, A\&AS, 125, 149

Feigelson, E. D., \& Montmerle, Th. 1999, ARA\&A, 37, 363

Feigelson, E. D., \& DeCampli, W. M. 1981, ApJ, 243, L89

Güdel, M., Audard, M., Briggs, K., et al. 2001, A\&A, 365, L336

Herczeg, G. J., Linsky, J. L., Valenti, J. A., Johns-Krull, Ch. M., \& Wood, B. E. 2002, ApJ, 572, 310

Hoff, W., Henning, Th., \& Pfau, W. 1998, A\&A, 336, 242

Kastner, J. H., Zuckerman, B., Weintraub, D. A., \& Forveille, T. 1997, Science, 277, 67

Kastner, J. H., Huenemoerder, D. P., Schulz, N. S., \& Weintraub, D. A. 1999, ApJ, 525, 837

Kastner, J. H., Huenemoerder, D. P., Schulz, N. S., \& Canizares, C. R. 2002, ApJ, 567, 434
Kenyon, S., \& Hartmann, L. 1995, ApJS, 101, 117

Lamzin, S. A. 1999, Astr. Lett., 25, 7

Lamzin, S. A. 1995, A\&A, 295, L20

Mewe, R., Gronenschild, E. H. B. M., \& van den Oord, G. H. J. 1985, A\&AS, 62, 197

Mewe, R., Kaastra, J. S., Schrijver, C. J., van den Oord, G. H. J., \& Alkemade, F. J. M. 1995, A\&A, 296, 477

Montmerle, T., Koch-Miramond, L., Falgarone, E., \& Grindlay, J. E. 1983, ApJ, 269, 182

Morrison, R., \& McCammon, D. 1983, ApJ, 270, 119

Muzerolle, J., Calvet, N., Briceño, C., et al. 2002, ApJ, 535, L47

Ness, J.-U., Mewe, R., Schmitt, J. H. M. M., et al. 2001, A\&A, 367, 282

Ness, J.-U., Schmitt, J. H. M. M., Burwitz, V., et al. 2002, A\&A, 394, 911

Ness, J.-U., \& Wichmann, R. 2002, AN, 323, 129

Neuhäuser, R., Sterzik, M. F., Schmitt, J. H. M. M., Wichmann, R., \& Krautter, J. 1995, A\&A, 297, 391

Phillips, K. J. H., Mewe, R., Harra-Murnion, L. K., et al. 1999, A\&AS, 138,381

Porquet, D., Mewe, R., Dubau, J., Raassen, A. J. J., \& Kaastra, J. S. 2001, A\&A, 376, 1113

Rucinski, S. M., \& Krautter, J. 1983, A\&A, 121, 217

Schmitt, J. H. M. M., \& Ness, J.-U. 2004, A\&A, 415, 1099

Schmitt, J. H. M. M., \& Ness, J.-U. 2002, A\&A, 388, L13

Stelzer, B., Neuhäuser, R., \& Hambaryan, V. 2000, A\&A, 356, 949

Stelzer, B., \& Neuhäuser, R. 2001a, A\&A, 377, 538

Stelzer, B., \& Neuhäuser, R. 2001b, in Proc. Young Stars near Earth: Progress and Prospects, March 28-30 2001, Ames Research Center, CA, USA, ed. R. Jayawardhana, \& T. Greene, ASP Conf. Ser., 244, 153

Stelzer, B., Burwitz, V., Audard, M., et al. 2002, A\&A, 392, 585

Walter, F. M., \& Kuhi, L. V. 1981, ApJ, 250, 254

Weinberger, A. J., Becklin, E. E., Schneider, G., Chiang, E. I., \& Lowrance, P. J. 2002, ApJ, 566, 409

Wichmann, R., Bastian, U., Krautter, J., Jankovics, I., \& Rucinski, S. M. 1998, MNRAS, 301, L39

Wichmann, R., Krautter, J., Schmitt, J. H. M. M., et al. 1996, A\&AS, 312,439 